\title{
FTO-associated obesity risk is linked to brain food responses via modulation of ghrelin levels
}

The obesity risk gene FTO exerts its proadipogenic effects via epitranscriptomic regulation of ghrelin mRNA, show new findings. "Our work has shown that in response to eating a meal, people with the FTO obesity-risk variant fail to suppress their hunger and circulating ghrelin levels," summarises senior researcher Rachel L. Batterham from University College London, UK.

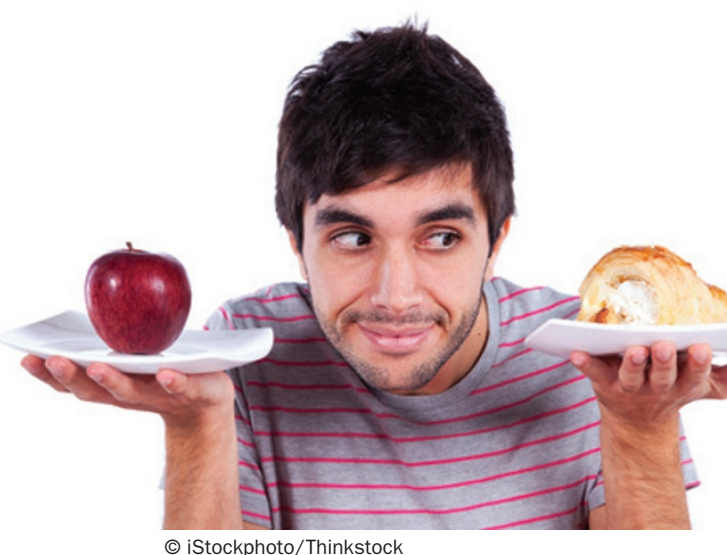

(๔) iStockphoto/Thinkstock
Single nucleotide polymorphisms within the FTO gene, which encodes an mRNA-modifying demethylase, pose a 1.7-fold increased risk of developing obesity in homozygous individuals. In accordance with this association, carriers of the risk allele were previously reported to have increased food intake and a preference for high-fat foods.

The investigators compared nonobese, homozygous carriers of the FTO obesityrisk variant with adiposity-matched homozygous carriers of a low-risk allele. After a test meal, high-risk probands showed impaired reduction of appetitestimulating ghrelin levels and rated high-calorie food images as more appealing than did control individuals. Specifically, postprandial functional MRI revealed increased neuronal activity in brain regions controlling appetite, reward and motivation, a phenotype very similar to the actions of exogenously administered active ghrelin.
In cell-based assays, FTO overexpression resulted in higher ghrelin mRNA expression and total levels of active ghrelin compared with controls. This finding was paralleled by reduced methylation of ghrelin mRNA owing to demethylation catalysed by FTO, providing a direct link between FTO risk alleles and ghrelin action.

The authors suggest that the FTO genotype alters brain responsivity to circulating ghrelin levels. The identified link could be the basis for "strategies aimed at modulating the ghrelin system to help prevent people with the obesity-risk FTO variant from becoming overweight, and also help overweight or obese people lose weight," concludes Batterham.

\section{Leah Eissing}

Original article Karra, E. et al. A link between FTO, ghrelin and impaired brain food-cue responsivity. J. Clin. Invest. doi:10.1172/JCl44403 